\title{
STUDY ON ENHANCEMENT OF OSSEOINTEGRATION OF THE BIO-ACTIVE TITANIUM IMPLANT BY LOW INTENSIVE ULTRASOUND WAVE
}

\author{
( PART II : IN VIVO EXPERIMENT) \\ Masanori Kobayahsi ${ }^{1}$, Masafumi Shiraishi ${ }^{2}$, Mitsuo Kinoshita ${ }^{2}$ \\ ${ }^{1}$ Department of Physical Therapy, \\ Takarazuka University of Medical and Health Care, Hyogo, Japan. \\ ${ }^{2}$ Department of Orthopedics Surgery, \\ Osaka Medical school, Osaka, Japan
}

\begin{abstract}
We have already reported the in vitro effect of the ultrasound wave stimulation for the hydroxyapatite-like crystalline formation on bioactive pure titanium surface in previous study ( Part I ), which indicate the clinical advantage to accelerate the bone-binding to bioactive material.

In this study ( Part II ), animal experiment was performed in order to investigate the effect of ultrasound waving in vivo. Bioactive pure titanium was processed by the chemical treatment and the heat treatment, implanted to the rabbits femur, and undertaken the irradiation of ultrasound waving for planned timeperiods. The titanium specimens taken from sacrificed rabbit was assessed by using Scanning Electron Microscope (SEM), histological observation and mechanical testing. As a result, the bone-bonding condition of titanium sample of the ultrasound waving radiation group was significantly excellent as compared with the non-ultrasound pulse group. These findings supported the result of previous in vitro study and suggested that the ultrasound waving had clinical potential to improve the osteointegration, bone-bonding ability for bioactive titanium implant.
\end{abstract}

\section{KEYWORDS}

Bio-active titanium, The low-intensity pulsed ultrasound waves, Osseointegration, Animal experiment

\section{INTRODUCTION}

It is well known that titanium and its alloys have excellent biocompatibility and mechanical properties, and have been widely used as medical implants under load-bearing conditions for dental and orthopedic fields. Since Osseointegration was first defined by Brånemark et al., these metals has been required the direct bone-bonding ability such as this osseointegration, osteoconductivity from the view of postoperative therapeutics, bio-active titanium with a new type of the surface treatment has been developed [1-6].

DOI: 10.5121/ijbes.2018.5101 
International Journal of Biomedical Engineering and Science (IJBES), Vol. 5, No. 1, January 2018

It has been already shown that this bioactive-titanium surface exhibits bioconductive and osseoconductive properties by many researches in the EU, USA and Japan. The results suggested the possibility of reducing the healing period by using titanium dental implants, however, the success rate of the further rapid and strong osseointegration of bioactive titanium implant has been required for the clinical early or immediate loading of an implant .

As reported in previous simulated body fluid (SBF) soaking test, the ultrasound waving stimulation on bioactive titanium surface showed the enhancement of the bone like hydroxyapatite crystalline formation on the surface.

The purpose of this study was to investigate whether the low-intensity pulsed ultrasound waves could accelerate the Osseointegration ability of bio-active titanium in vivo.

\section{MATERials}

In the present study, pure titanium was used as an implant for animal experiment because pure titanium specimens showed higher osseointegration effect by the ultrasound pulsed radiating in previous SBF soaking study.

The bioactive pure titanium implant was prepared according to a following procedure. Briefly, samples of pure titanium ( $99.9 \% \mathrm{Ti}$; Daido steel Co. Japan) were cut into two kinds of sizes, i.e. $3 \mathrm{~mm} \times 3 \mathrm{~mm} \times 3 \mathrm{~mm}$ cubic and $3 \mathrm{~mm} \times 3 \mathrm{~mm} \times 9 \mathrm{~mm}$ cuboid dice, polished and chemically treated with $\mathrm{NaOH}$ solution (concentration : $2 \mathrm{M} / \mathrm{L}$ ) for 2 hours reaction time. After that, these samples were heated at $600{ }^{\circ} \mathrm{C}$ for 1 hour in an electric furnace and then cooled to room temperature in the furnace for 24 hours to modify the surface. These chemical procedures were same as that in previous SBF soaking test.

\section{METHODS}

\subsection{In vivo Experiment ( Animal Experiment )}

An experimental in vivo study using the rabbit was done in order to evaluate the effect of ultrasound waving radiation on the osseointegration of the bioactive titanium.

For this experiment, the bilateral femoral greater trochanter of eight mature female rabbits ( body weight 2.5-3.0 Kg ) were used. Under intravenous anesthesia with Nembutal (2-3ml), the operation was performed using aseptic procedures. The implantation site of femur was exposed through a longitudinal incision on the lateral side of hip joint, and blunt dissection to fascia lata. The periosteum was elevated at the implant insertion area, and a bone hole was made using a drill and file, and processed into a $3 \mathrm{~mm} \times 3 \mathrm{~mm} \times 3 \mathrm{~mm}$ deep cuboid cavity to insert one titanium specimen in each femoral site.

And then, Four pairs of $3 \mathrm{~mm} \times 3 \mathrm{~mm} \times 3 \mathrm{~mm}$ cubic titanium specimens for histological evaluation, four pairs of $3 \mathrm{~mm} \times 3 \mathrm{~mm} \times 9 \mathrm{~mm}$ cuboid titanium for mechanical bonding strength measurement were implanted respectively. After implanting the specimen, surgery area were washed, the overlying soft tissue was closed in layers. Fig.1 shows the schema of this experimental rabbits femur. 


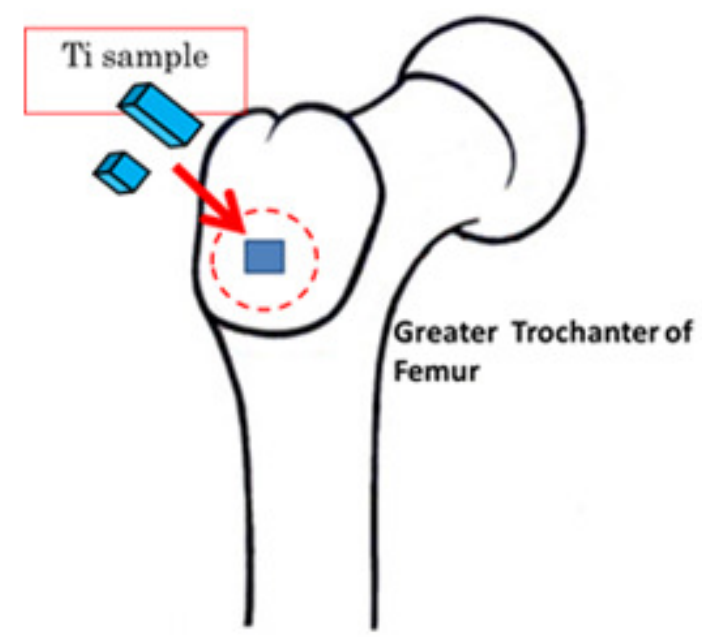

Figure.1 The schematic diagram of this surgical experiment

After operation, one side of femur of rabbits was radiated with the ultrasound waving by using Sonic Accelerated Fracture Healing System (SAFHS; Smith \& Nephew, Memphis, TN, USA ; Teijin Pharma, Tokyo, Japan ) as an USW group, another side was not radiated as a control group.

\subsection{Analysis}

All rabbits were reared in cages with no postoperative immobilization, and sacrificed at four weeks after operation to assess the titanium samples. All femurs with bioactive titanium implant were resected en bloc from rabbits, and soft tissue were removed, titanium implant sample was examined mechanically and histologically .

For histological observation, two pairs of femur were fixed in formalin, decalcified with PlankRycho solution, cut into sections, and stained with hematoxylin and eosin (H-E) staining. Two pairs of femurs with titanium sample were subjected to scanning electron microscopy (SEM), and four pairs of femurs were subjected to the mechanical pull-out testing for evaluation of the titanium - bone bonding strength, after that, the titanium sample surface were analyzed by energy dispersive spectroscopy (EDS) and X-ray diffraction (XRD).

All the above manipulations in the experimental process were in conformity with the Guideline for Animal Experiments, Osaka Medical College of Japan.

\section{RESULTS}

\subsection{Macro appearance of the animal specimens}

Fig. 2 shows the macro appearance of implant site on rabbits femur. Neither inflammation nor infection sign was observed in this area. All titanium implants were fixed in the femoral cortical bone. 
International Journal of Biomedical Engineering and Science (IJBES), Vol. 5, No. 1, January 2018

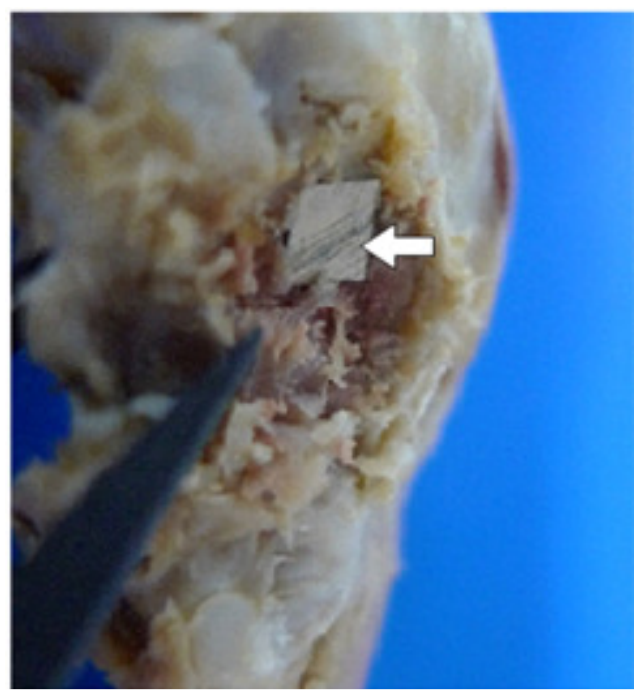

Figure. 2 Macro appearance of rabbits femoral bone. Arrow $(\rightarrow)$ shows the titanium sample implant

\subsection{Micro analysis}

\subsubsection{SEM observation}

Fig.3 shows the microappearance of implant onto femur by SEM. The interface between the titanium sample and femoral bone in Ultrasound waving group (USW) exhibited more high dense bone tissue and more narrow dead space as compared with control group ( non-irradiation of ultrasound waving ).

(a)

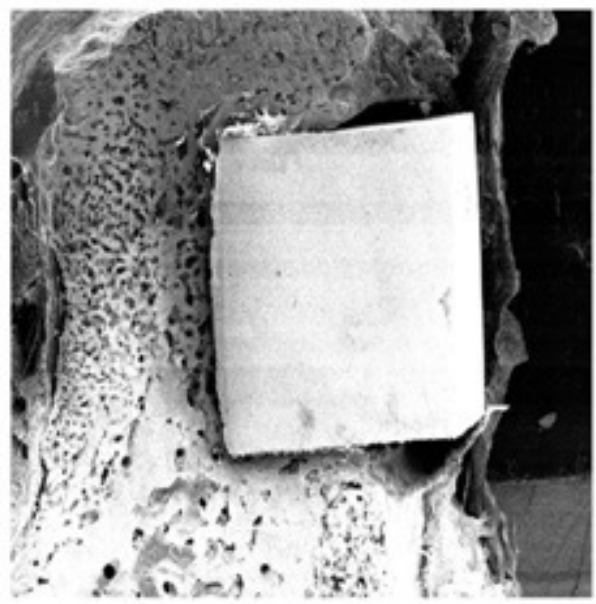

(b)

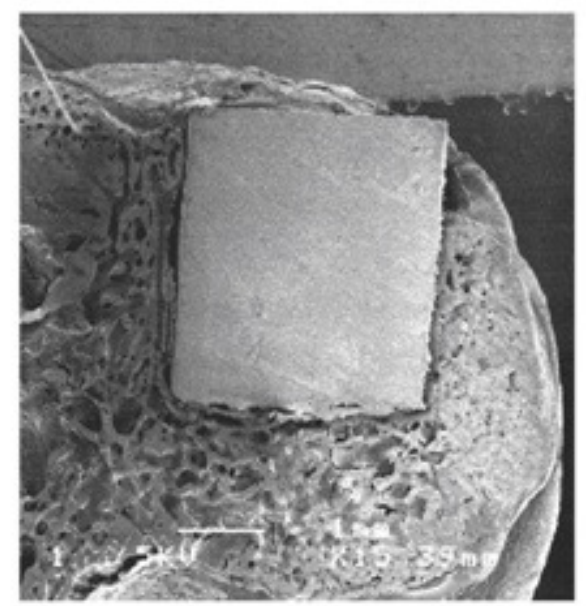

(b) Ultrasound waving group

Furthermore, as shown in Fig.4, some direct contact like areas of titanium and bone tissue were observed in high magnification appearance of Ultrasound waving group . 


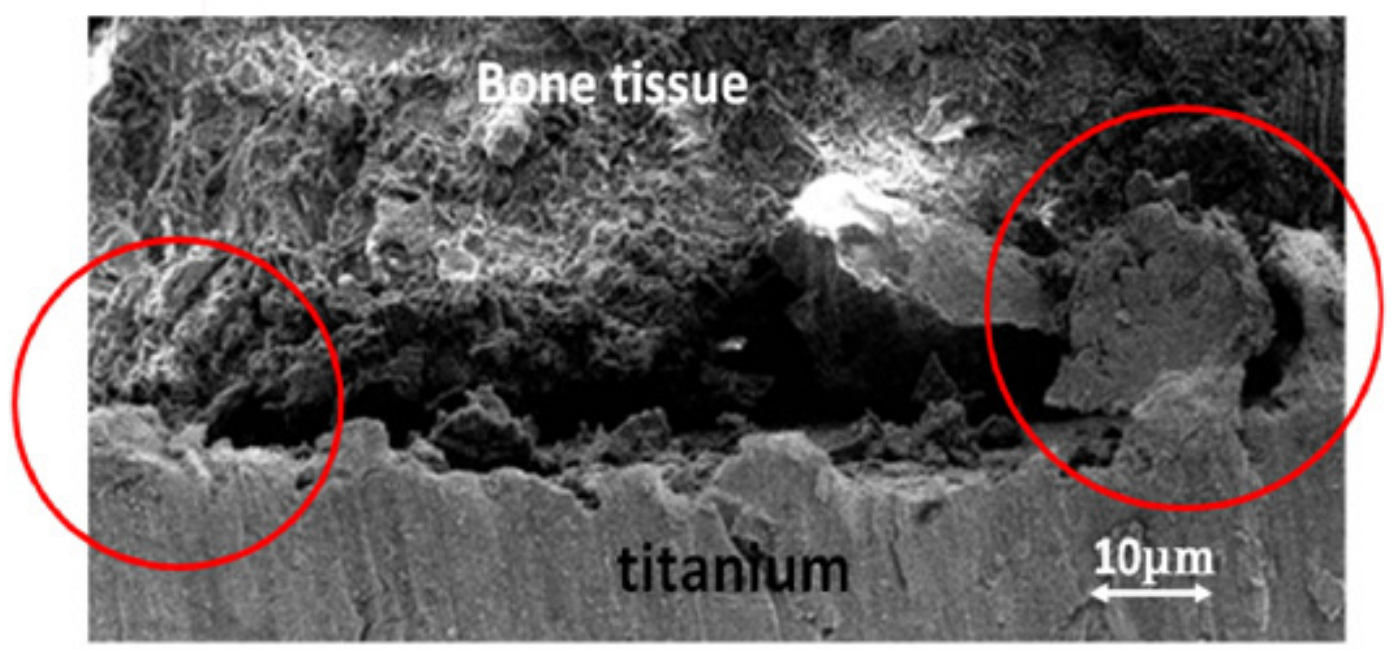

Figure.4 SEM image of the interface between the bone tissue and bioactive titanium sample of ultrasound waving group $(\times 3000)$

\subsubsection{Histological Observation}

Fig.5 shows the histological finding of implant onto femur bone tissue by H-E stain. This histological findings also exhibited more direct attachment areas as shown by arrow in the interface between the titanium sample and femoral bone in Ultrasound waving group as compared with control group ( non-irradiation of ultrasound waving )

In addition, the implant surrounding bone tissue also exhibited more rich genesis in Ultrasound waving group.

(a)
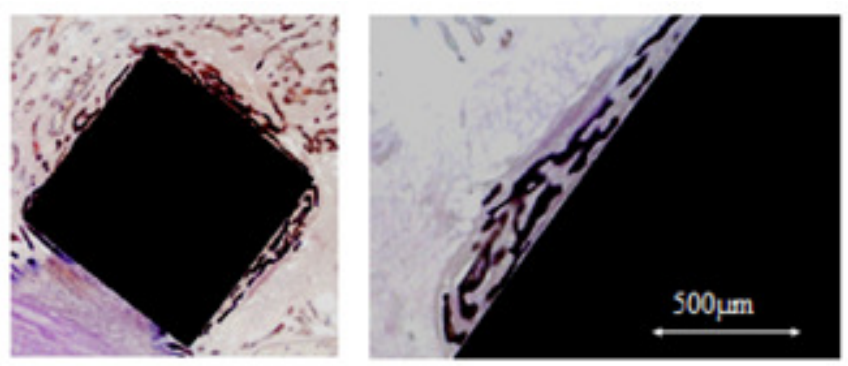

(b)
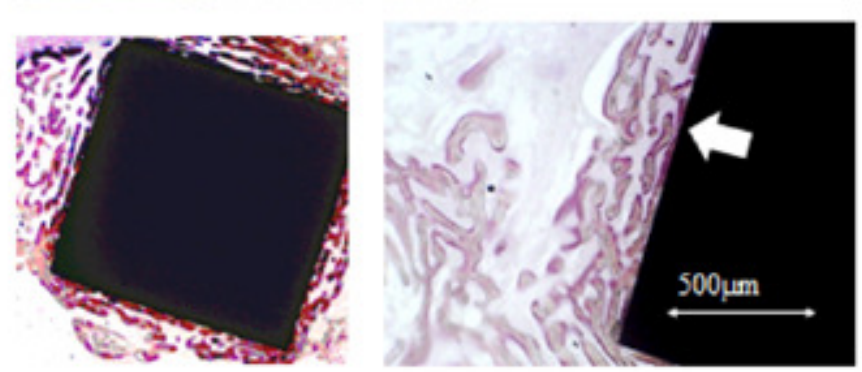

Figure.5 Histological appearance of the surface of bioactive titanium sample onto bone

(a) Control group (without ultrasound waving) (b) Ultrasound waving group 
International Journal of Biomedical Engineering and Science (IJBES), Vol. 5, No. 1, January 2018

\section{4..3 Mechanical test}

\subsubsection{Measurement of the bonding strength between the Ti implant and bone tissue}

Fig.6 shows the schema of the mechanical pull-out testing machine. The titanium implant was pulled out from femur bone by the grasping jig with $1 \mathrm{~mm} / \mathrm{sec}$ and measured the load-distance ( stress-strain ) curve.

(a)

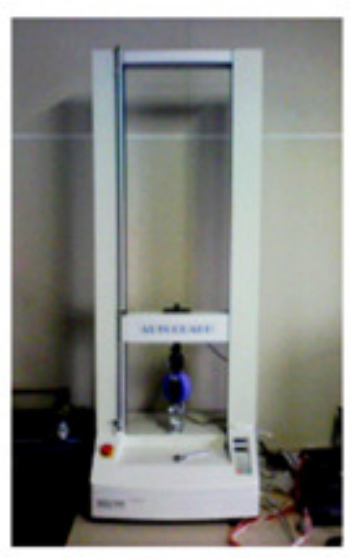

(b)

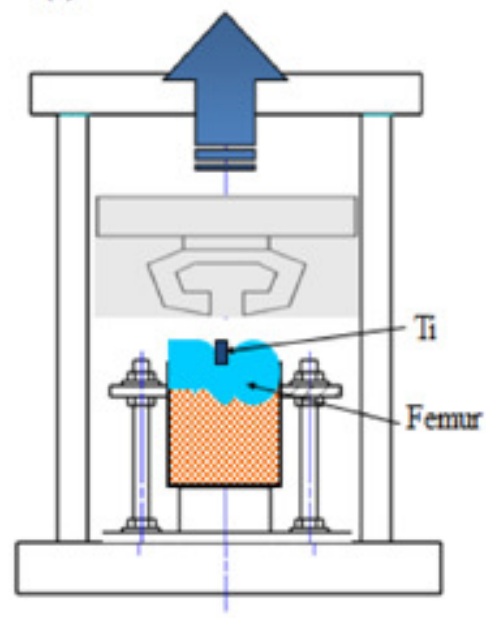

Figure.6 The mechanical pull-out test machine ( photograph and mechanical testing schema)

The results of mechanical test are shown in Fig.7. One sample data of Load-Displacement curves indicated higher bonding strength and mechanical toughness in USW group as compared with control group (Fig,7 (a)), total bonding strength values of two groups also suggested the significant difference between the results of the USW and control group ( Fig,(b)).

(a)

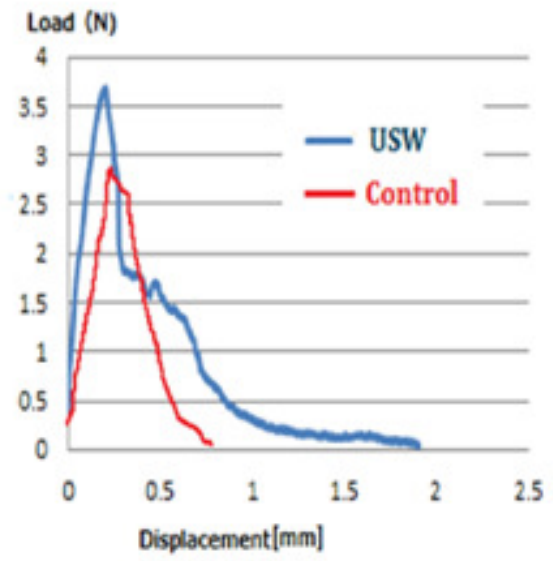

(b)

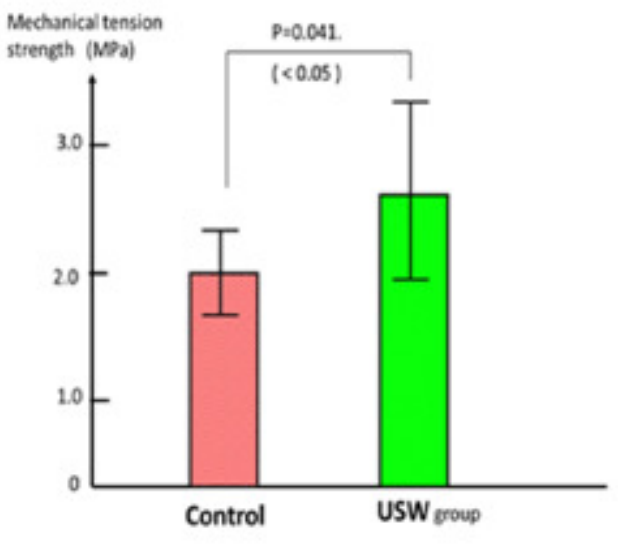

Figure.7 The results of pull-out tests (a) The load-displacement relation curves (b) Bone-titanium bonding strength 
International Journal of Biomedical Engineering and Science (IJBES), Vol. 5, No. 1, January 2018

\subsection{2 macro and micro analysis of the implant surfaces}

Fig 8 shows the macro appearance of implanted titanium sample of USW group after mechanical pull out test. The surface titanium sample has some attached bone segments of femur, the amount and area of adhered bone segment were more rich in the ultrasound waving radiated titanium surface as compared with the control group.

The surfaces of bioactive titanium implants used in the mechanical pull-out testing were observed by SEM and analysed by energy dispersive spectroscopy (EDS) in addition.

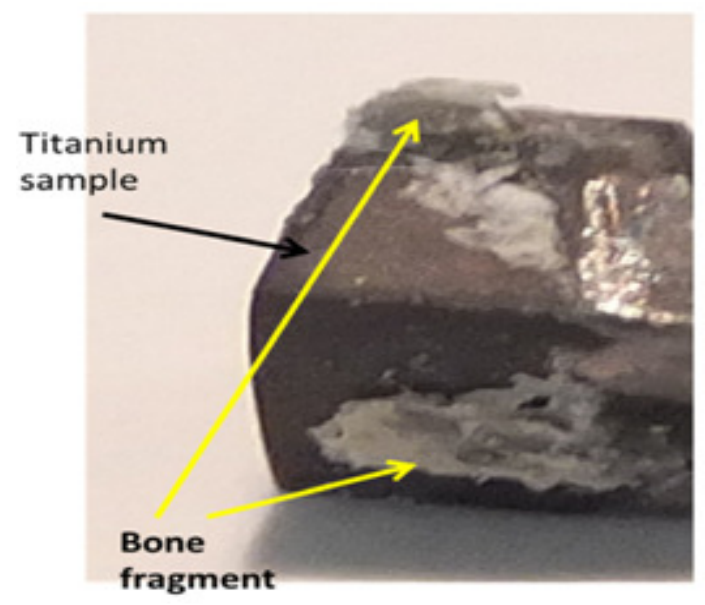

Figure.8 Macro appearance of the bioactive titanium sample after the pulled-out tests

Fig. 9 shows the SEM images of each titanium surfaces of control and ultrasound waving group. These images exhibited the precipitation layer on the surface, which is more rich covered on ultrasound waving group sample (b) as compared with (a) control group. As shown in Fig.10, this precipitation indicated the composition of rich phosphate $(\mathrm{P})$ and calcium $(\mathrm{Ca})$ as well as bone tissue according to EDS analysis.

(a)

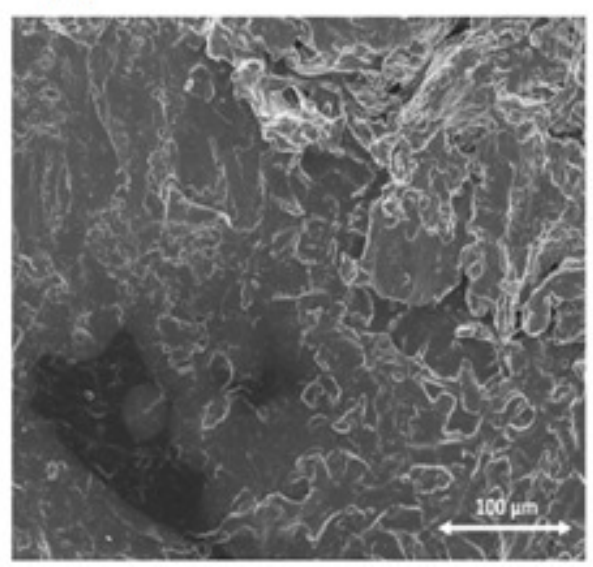

(b)

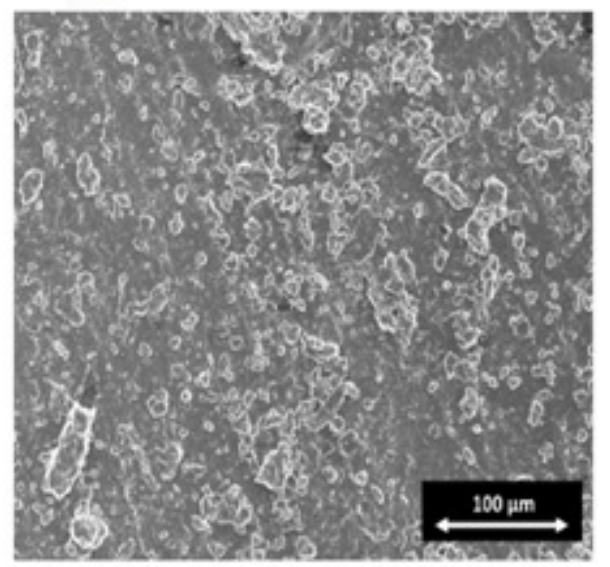

Figure.9 SEM image of the bioactive titanium sample surface after the pulled-out tests
(a) Control group
(b) Ultrasound waving group
$(\times 500)$ 


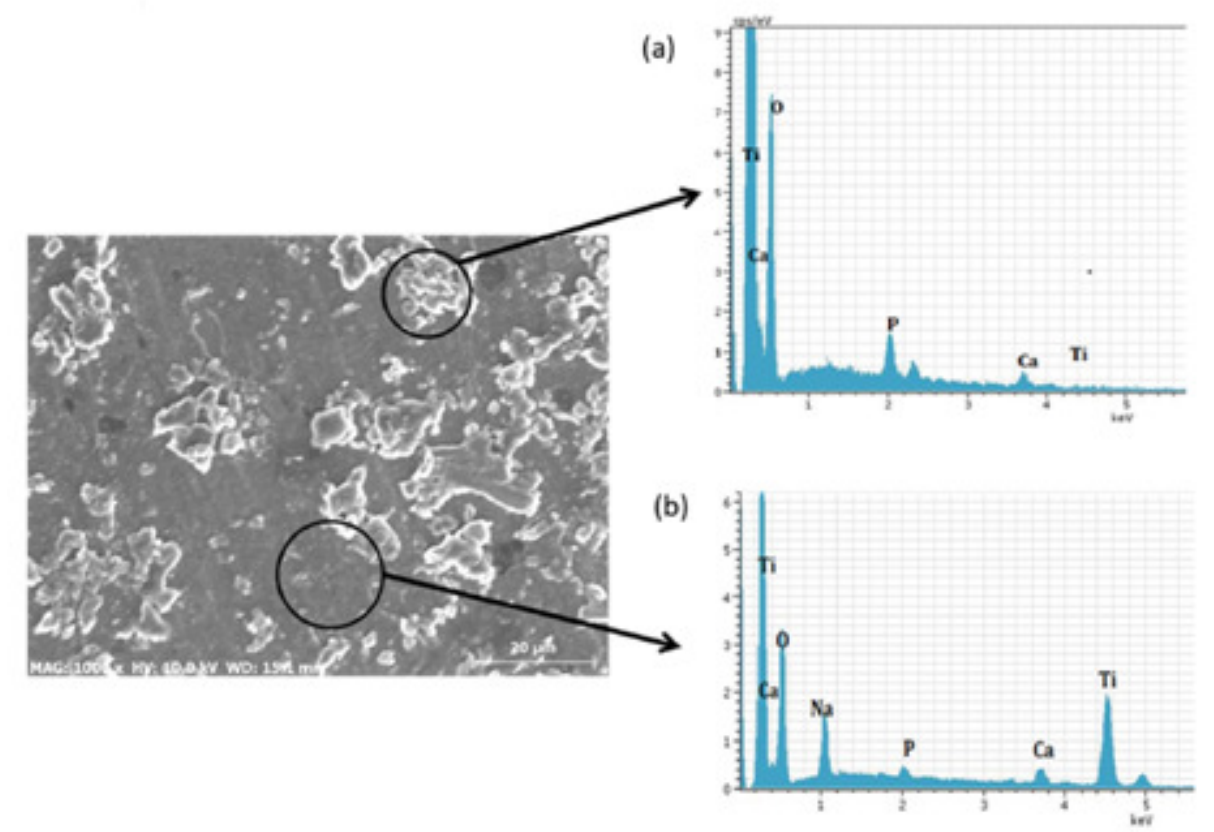

Figure.10 EDS analysis of bioactive titanium surface implanted into femur for 2 weeks $\begin{array}{ll}\text { (a) The precipitation area } & \text { (b) The Ti based surface }\end{array}$

These findings indicated the bone - bonding condition on the implant surface broken out from bone tissue, more rich calcium-phosphate crystal layer in the specimen of ultrasound waving group could associate with the enhancement of osseointegration by Ultrasound waving.

\section{DISCUSSION}

The simulation method using SBF soaking in this study is well known as a in vitro assay for evaluation of bone-bonding ability of biomaterials, and has been already used widely for the study regarding the osteoconductivity [3-7].

In our previous report, using same experimental methods, the increasing of calcium-phosphate precipitation including partial hydroxyapatite crystalline by ultrasound wave irradiation was confirmed. From morphological observation by SEM and chemical analysis about the precipitation substrate on the titanium surface soaked in simulated body fluid, we speculate that the ultrasound waves promote the nucleation and crystal growth of calcium phosphate compounds at the initial state of the crystallization process. It might be that the partial concentration and interatomic potential of the $\mathrm{Ca}$ and $\mathrm{PO} 4$ ions in SBF can be changed by ultrasound micro vibration, and accelerated to the nucleation and deposition of ions, resulting in the crystallization of calcium phosphate on activated titanium surface layer. Cavitation by ultrasound waves might also contribute to formation of the apatite layer by means of the microfracture of calciumphosphate and the circulation of new $\mathrm{Ca}$ and PO4 ions of SBF on the titanium surface. These environmental changes will accelerate further changes of interatomic potential of surrounding atoms, and deteriorate the thermal stability of calcium-phosphate phase versus $\mathrm{Ca}$ and PO4 ions. 
International Journal of Biomedical Engineering and Science (IJBES), Vol. 5, No. 1, January 2018

Following previous study, the results of animal experiments using rabbits also supported the effect of ultrasound wave irradiation for enhance of hydroxyapatite crystallization on the bioactive titanium modified surface and reinforcement of bone-bonding strength.

In vivo, the mechanism of the ossointegration enhancement by ultrasound wave irradiation is more complicate and difficult to elucidate because of the biological osteogenesis function by osteoblast and fibroblast cells etc. Though low-intensive pulsed ultrasound waves have been shown to enhance the osteogenesis and are widely used as clinical treatment for complicated bone fracture in the orthopaedics surgery field [8], little is known about the mechanism of action of ultrasound. During the experiments and investigations of this subject, many of these studies have reported biological mechanisms based on the metabolic reaction induced by various activated osteogenic cells by mechanical stress [9-12].

However, considering the clinical efficacy regarding the result of the mechanical bonding strength between bone tissue and titanium implant in this study, this low intensive pulsed ultrasound waving is strongly expected as an excellent application for the enhance of osseointegration of bio-active titanium implant in dental surgery fields. Hereafter, further assessment regarding the optimum conditions such as frequency and intensity of vibration of ultrasound waving for acceleration of hydroxyapatite crystallization on the modified titanium surface will be necessary.

\section{REFERENCES}

[1] Brånemark, P.I.; Hansson, B.O.; Adell, R.; Breine, U.; Lindström, J.; Hallén, O. \& Ohman, A. (1997) "Osseointegrated implants in the treatment of the edentulous jaw. Experience from a 10-year period." Scandinavian Journal of Plastic and Reconstructive Surgery Supplementum, Vol.16, pp 1-132.

[2] Brånemark, P.I.. (1983) "Osseointegration and its experimental background." Journal of Prosthetic Dentistry, Vol.50, No.3, pp. 399-410

[3] Kuroda D, Okido M, (2007) "Surface modification of metallic implant harmonized with hard tissue." Kinzoku (Materials Science \& Technology). Vol. 277(2), pp 141-7. ( Japanese )

[4] Kokubo T, Yamaguchi S. Novel Bioactive Titanate Layers Formed on Ti Metal and Its Alloy by Chemical Treatments. Materials Vol. 3 (2010) pp.48-63

[5] Nishiguchi S, Fujibayashi S, Kim HM, Kokubo T, Nakamura T. Biology of alkali- and heat-treated titanium implants. Journal of Biomedical Material Research A Vol.67 (2003) pp.26-35

[6] Kim HM, Kokubo T . (2003). " Surface potential change in bioactive titanium metal during the process of apatite formation in simulated body fluid." Journal of Biomedical Material Research A. Vol.67, pp.1305-9.

[7] Kokubo T, Takamada H, (2006) "How useful is SBF in predicting in vivo bone bioactivity? " Biomaterials Vol. 27 pp 2907-15

[8] Mizuno K, Sumida M, Matsui J, Fujita M, Takigawa S, Kokubu T. (2000) “ Current topics on fracture treatment with ultrasound" Orthopaedic Surgery and Traumatology. Vol. 43 No.3, pp 213223. ( Japanese ) 
International Journal of Biomedical Engineering and Science (IJBES), Vol. 5, No. 1, January 2018

[9] Yang KH, Parvizi J, Wang SJ, Lewallen DG, Kinnick RR, Greenleaf JF, Bolander ME. (1996) “ Exposure to low-intensity ultrasound increases aggrecan gene expression in a rat femur fracture model." J.Orthop Res. Vol. 14, pp 802-809.

[10] Uglow MG, Peat RA, Hile MS, Bilston LE, Smith EJ, Little DG. (2003) “ Low-intensity ultrasound stimulation in distraction osteogenesis in rabbits." Cl.Orthop. Vol. 417, pp 303-312.

[11] Lorenzo Fassina, Enrica Saino, Maria Gabriella Cusella De Angelis, GiovanniMagenes, Francesco Benazzo, Livia Visai. (2010) " Low-Power Ultrasounds as a Tool to Culture Human Osteoblasts inside Cancellous Hydroxyapatite.” Bioinorganic Chemistry and Applications. pp 1-8.

[12] O'brien WD, (2007) “Ultrasound-biophysics mechanisms." Progress in biophysics and molecular biology. Vol. 93, pp 280-94. 